\title{
Structural Characterization of RF-Sputtered CZTS Thin Films
}

\author{
Mustapha K.A. ${ }^{1}$ Moreh A.U. ${ }^{2}$ Sulu H.T. ${ }^{3}$ \\ Research Scholar ${ }^{1,3}$, Doctor ${ }^{2}$ \\ Department of Physics \\ Usmanu Danfodiyo University \\ Sokoto, Sokoto State \\ Nigeria
}

\begin{abstract}
Polycrystalline CZTS thin films of thickness $45 \mathrm{~nm}, 90 \mathrm{~nm}, 140 \mathrm{~nm}$ and 180nm have been grown on the corning glass substrate by RF-magnetron sputtering at a substrate temperature of $1000 \mathrm{C}$ using a quaternary target. Structural characteristics of the grown thin films have been investigated using X-ray diffractometer (XRD). Detailed analysis of the XRD data has shown that the grown CZTS thin films have kesterite structure with preferred orientation along (112) plane which was observed to become stronger with an increase in film thickness, especially in the annealed samples. All the peaks observed in the XRD pattern have been accounted for kesterite structure, which shows the absence of additional phases such as elemental or binary or ternary systems in the grown film. The above observations show that the material under investigation is suitable for solar cell applications.
\end{abstract}

Key Words: XRD, CZTS, RF-Magnetron Sputtering.

\section{INTRODUCTION}

As human civilization continues to expand into the 21 st century, so do the challenges associated with global power production. In 2012, the world consumed an estimated $17.7 \mathrm{TW}\left(1.77 \times 10^{13} \mathrm{~W}\right)$ of power which is projected to increase $56 \%$ by $2040[1]$. Continuing to meet this growing power demand has prompted increasing concern over the limited supply and environmental impact of fossil fuels, which account for $85 \%$ of the world's energy production [2]. This has motivated a shift towards energy production from clean, renewable technologies such as wind, solar, and hydroelectric. Among these technologies, solar power has gained considerable attention for having the potential for terawatt (TW) level power production. With approximately 23,000 TW of solar energy arriving at the earth's surface every year, only a miniscule amount would need to be converted to supply the world's energy needs. Despite this potential, only $177 \mathrm{GW}$ of solar power was generated in 2014 signifying the need for further progress in photovoltaics(PV) research to increase the feasibility of solar power [3]. The entire world's energy demand can be met if we cover only $0.1 \%$ of the earth's surface with about $10 \%$ efficient solar cells. In spite of this great potential, energy derived from solar cells comprised of about $0.005 \%$ of the total energy produced in the U.S in 2010 [4]. The most commonly used solar panels are made from high purity, crystalline silicon. Processing silicon is expensive and increases the overall cost of production of the solar panels.

Thin film solar cells are emerging as an alternative technology to silicon-based solar cells. In these devices, thin $(\sim 1 \mu \mathrm{m})$ films of semiconductor materials such as cadmium telluride (CdTe), and copper indium gallium disellenide (CIGS) are used in lieu of a thick $(\sim 100 \mu \mathrm{m})$ silicon wafer. As the absorption coefficient of typical thin film absorber materials are $\sim 100$ times higher than crystalline silicon, a 100 times thinner layer of thin film materials can absorb an equivalent amount of energy as crystalline silicon. In addition, purity and crystal quality requirements on these thin film solar cell materials are less stringent than in crystalline silicon solar cells, as the grain boundaries in thin film materials enhance separation and transport, and permit only negligible recombination [5]. Thus, thin film solar cells are cheaper to produce than silicon solar cells. In essence, the consensus is 
that PVs will not be wide-spread until it is less expensive to fabricate, implement, and maintain such devices than to purchase mass produced power generated

from non-renewable sources like natural gas, coal, oil, or others. In the past few decades, there has been an ongoing search within the PV-research field for new materials and cell architectures that might circumvent the drawbacks to using silicon-based cells namely that the most efficient single-crystal silicon-based PVs require high production and high materials cost. Thin film PVs cells based on thin film semiconductors as opposed to bulk, necessary with single-crystal silicon cells - have been well studied utilizing different photo-absorbing materials. Copper-indium-gallium-selenide (CIGS) and cadmium telluride (CdTe) have both been extensively researched, and have been proved to be efficient enough to sell publicly via start up companies like First Solar (CdTe) [6] or Solar Frontier (CIGS) [7]. These materials, however, have significant drawbacks pertaining to mass market sustainability and environmental impact. Indium, in particular, is undesirable to use in a mass market product due to its scarcity on earth and its subsequent high cost, making this technology unsustainable for a large market [8].

Cadmium is a known human carcinogen, making its widespread commercial use undesirable due to possible negative health and environmental effects. Relatively recently, in the past 25 years, copper-zinc tin sulphide $-\mathrm{Cu}_{2} \mathrm{ZnSnS}_{4}(\mathrm{CZTS})-$ has been investigated as a thin film photo absorber for use in PV devices. This material is desirable due to the component elements' lowcost and Earth-abundance. The band gap of CZTS is between 1-1.5eV, and the material has an absorption coefficient on the order of $10^{4}$, both desirable characteristics for use as a photo-absorber in a PV [9]. The primary advantage of using CZTS is that it offsets the drawbacks of silicon, CIGS, and CdTe technologies. Copper, zinc, tin, and sulfur are all relatively inexpensive, earth abundant, and environmentally friendly.

\subsection{EXPERIMENTAL DETAILS}

\subsection{Substrate preparation and cleaning}

All depositions were carried out in a class 100 clean room. Before the deposition, the substrates were kept in a dilute chemical detergent (a detergent solution used in the laboratory to solubilise biological macromolecules such as proteins) solution at $100^{\circ} \mathrm{C}$ in ultrasonic bath for 10 minutes to remove oils and protein molecules and rinsed with double distilled water to remove possible left detergent contaminants. To remove organic contaminants, the substrates were boiled in dilute hydrogen peroxide $\left(\mathrm{H}_{2} \mathrm{O}_{2}\right)$ solution for 15 minutes. The same solutions were put into the ultrasonic bath. The substrates were extracted from the bath and rinsed with distilled water and later dried with $4 \mathrm{~N}$ nitrogen gas before being introduced into the sputtering chamber.

\subsection{Thin film deposition}

The deposition begins with loading the target and securing the glass substrate(s) to the substrate holder. The sputtering machine consists of a stainless steel chamber with a cover that moves vertically (up and down) and three sputter magnetron guns of $2 \mathrm{~cm}$ in diameter each located at the bottom of the chamber. The chamber is normally evacuated and then partially filled with nitrogen gas to $0.13-0.27 \times 10^{3} \mathrm{~Pa}$ so as to keep it clean. The target is a single disk composed of CZTS of $99.99 \%$ purity having a diameter of about $2 \mathrm{~cm}$ and a thickness of about $0.25 \mathrm{~cm}$. Sputtering was performed on a corning glass substrate under a constant flow of argon gas $\left(99.99 \%\right.$ purity) with a fixed target/substrate distance of $\sim 7 \mathrm{~cm}$. The base pressure was around $0.13 \mathrm{x} 10^{3} \mathrm{~Pa}$. The thickness of the deposition films were 45, 95,140, and $180 \mathrm{~nm}$. All the films were deposited at a substrate temperature of $100^{\circ} \mathrm{C}$.

Before the samples were characterized to analyze their various properties, heat at a temperature of $450^{\circ} \mathrm{C}$ was applied to four of the samples i.e., there are two batches of four samples each, making a total of eight samples of various thickness as-deposited and annealed. The ramp rate was $10^{\circ} \mathrm{C}$ per minute and the dwelling time was 1 hour. The samples were annealed under nitrogen gas flowing at $10 \mathrm{sccm}$ per minute. The purity of the nitrogen gas was $99.99 \%$.

\subsection{Characterization}

The X-ray tube was operated at a voltage of $45 \mathrm{kV}$ and a current of $40 \mathrm{~mA}$. XRD patterns were recorded in the range of $20^{0}-65^{0}$ with a scan speed of $2^{\circ} \mathrm{min}$, step size of $0.2000^{\circ}$ and scan step time of 1.10 seconds for all deposited thin films. In the Diffractometer, the X-ray source was in fixed position and the sample stage and the detector rotated simultaneously in the same plane, along the same axis (Diffractometer axis) perpendicular to the figure and located at the center of the sample stage. The sample was rotated and the detector followed the sample motion in the same direction around the Diffractometer axis by twice the speed of the sample. The angular positions and intensities of the resultant diffracted peaks of radiation from the planes parallel to the substrate surface were detected and recorded. By plotting the angular positions (i.e.2 $\theta$ ) and intensities of the resultant diffracted peaks of radiation, a pattern characteristic of the sample was obtained. The XRD diffractograms obtained for the samples were compared with XRD patterns of well-known material (nearly 62,000 different diffraction patterns) structures 
supplied by ICDD (International Centre for Diffraction Data) cards and detailed knowledge about the structure, phase composition and degree of crystallinity was obtained.

ICDD is an organization that supplies a database which consists of XRD spectra of organic and inorganic materials. It is supplied generally by manufacturer of diffraction systems. Based on the analysis of XRD patterns, valuable knowledge about the structure of CZTS thin films was obtained. And, with the help of measured full width at half maximum (FWHM) of preferred orientation, the grain sizes were calculated through the Scherer's formula

$$
\mathrm{t}=\frac{0.9 \lambda}{B \cos \theta}
$$

where $t$ is crystal depth, $\mathrm{B}$ is full width at half maximum, $\theta$ is Bragg's angle and $\lambda$ is thee wavelength of incident $\mathrm{X}$-rays.

\section{Determination of dislocation density $\delta$}

The dislocation density $(\delta)$, defined as the length of dislocation lines per unit volume, is the measure of the amount of defects in a crystal. Dislocations are imperfection in a crystal associated with misregistry of the lattice in one part of the crystal with respect to another part. Dislocations are not equilibrium imperfections. In fact growth mechanism involving dislocation is a matter of importance. The dislocation density of thin films were calculated by employing the relation [10].

$$
\delta=\frac{n}{D^{2}}
$$

Where $\mathrm{n}$, is a factor, which equals unity giving minimum dislocation density, $\delta$ and $\mathrm{D}$ is the grain size.

\section{Determination of Micro Strain $\varepsilon$}

The origin of strain is related to lattice 'misfit' who in turn depends upon the growing conditions of the films. The micro strain ( $\varepsilon$ ) developed in thin films was calculated from the relation [11].

$$
\varepsilon=\frac{\beta \cos \theta}{4}
$$

Where $\beta=$ FWHM (Full width at half maximum)

\section{Determination of Lattice Parameters $a$ and $c$}

The lattice parameters a and $\mathbf{c}$ value for tetragonal crystallographic system can be calculated from the following equation using $h k l$ parameter and the inter planer spacing $\mathbf{d}$

$$
\frac{1}{d^{2}}=\frac{h^{2}+k^{2}}{a^{2}}+\frac{l^{2}}{c^{2}}
$$

\subsection{RESULTS AND DISCUSSION}

The XRD patterns of CZTS grown at substrate temperature of $100^{\circ} \mathrm{C}$ and various thicknesses are shown in figure 4.1 (a-d).

Figure 4.1(a) shows the XRD patterns of CZTS thin films deposited at a thickness of 45nm. The as-deposited sample is completely amorphous as no peak is evident in the XRD pattern. However, after annealing the sample at a temperature of $450{ }^{\circ} \mathrm{C}$, low intensity peaks were observed around $28^{\circ}$ and $48^{\circ}$ of $2 \theta$ values respectively corresponding to (112) and (220) diffraction planes of kesterite CZTS. Similar observation was made by Tanaka et al., 2010. The reflections from these planes are recognized as characteristics of $\mathrm{Cu}_{2} \mathrm{ZnS}_{4}$ with tetragonal Kesterite structure by the joint committee on powder diffraction standards (JCPDS Card no. 26-0575). 


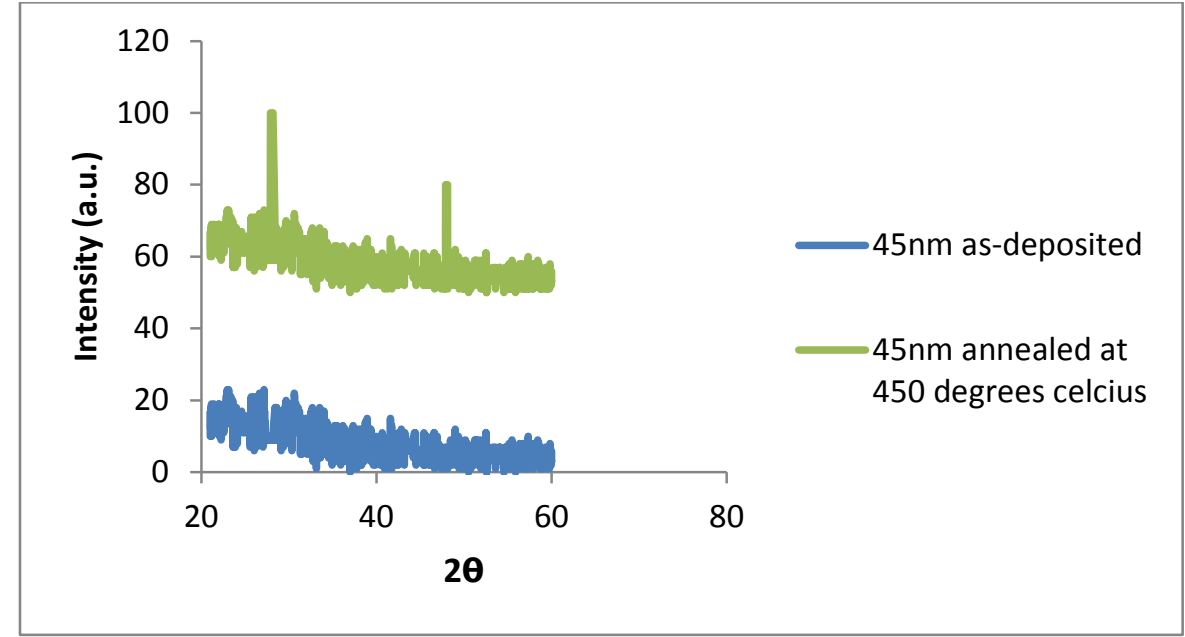

Figure 4.1(a): XRD pattern of CZTS thin film deposited at 45nm

The peaks for the samples deposited at $90 \mathrm{~nm}$ and $140 \mathrm{~nm}$ are similar to those deposited at $45 \mathrm{~nm}$ but with a higher intensity (fig $4.1 \mathrm{~b}$ and fig $4.1 \mathrm{c}$ ). The choice of annealing temperature of $450^{\circ} \mathrm{C}$ is responsible for the absence of secondary phases since annealing at lower temperatures causes formation of secondary phases and annealing at higher temperatures causes melting and subsequent evaporation of the film [12].

Figure 4.1(d) shows the XRD pattern of CZTS thin film deposited at film thickness of $180 \mathrm{~nm}$. In the as-deposited sample, the broad hump ranging from $20^{\circ}$ and $28^{\circ}$ corresponds to the mixed amorphous and crystalline nature having short range periodicity of mixed ternary and quaternary phases of CuZnS or CZTS materials. Upon heat treatment (annealing at $450^{\circ} \mathrm{C}$ ), the broad hump disappeared and the peaks exhibited

at about $28^{\circ}$ and $48^{\circ}$ respectively corresponds to (112) and (220) planes of tetragonal kesterite structure (JCPDS Card no. 260575). The transfer of one phase to another phase depends on the formation enthalpy of the particular phase [13].

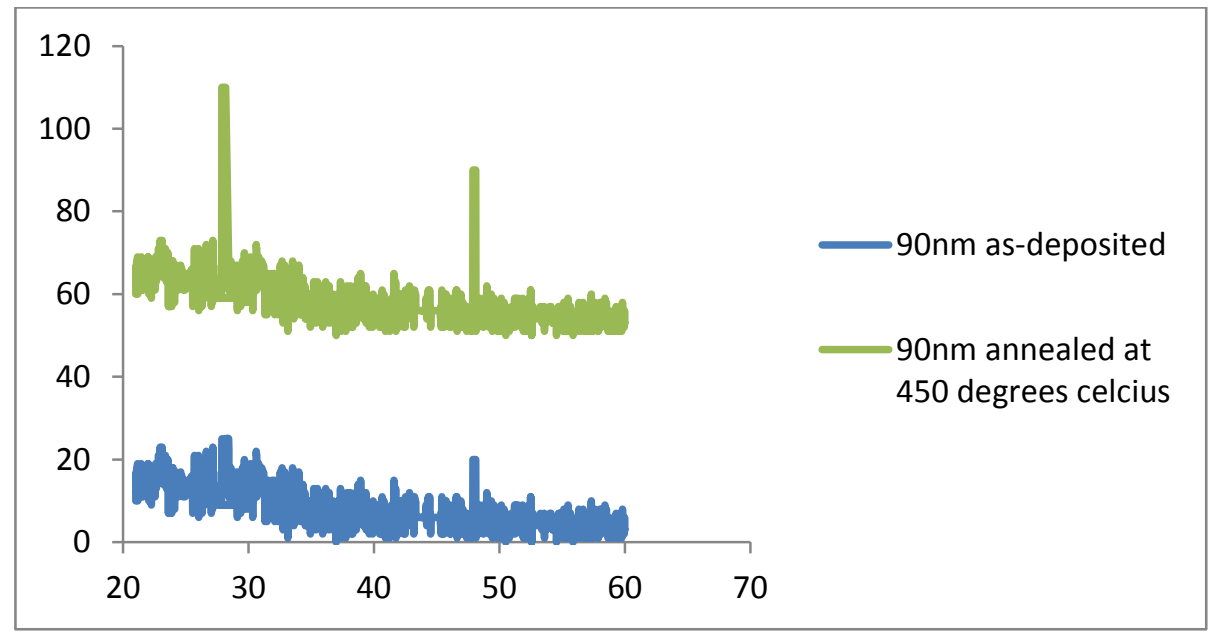

Figure 4.1(b): XRD pattern of CZTS thin film deposited at 90nm 


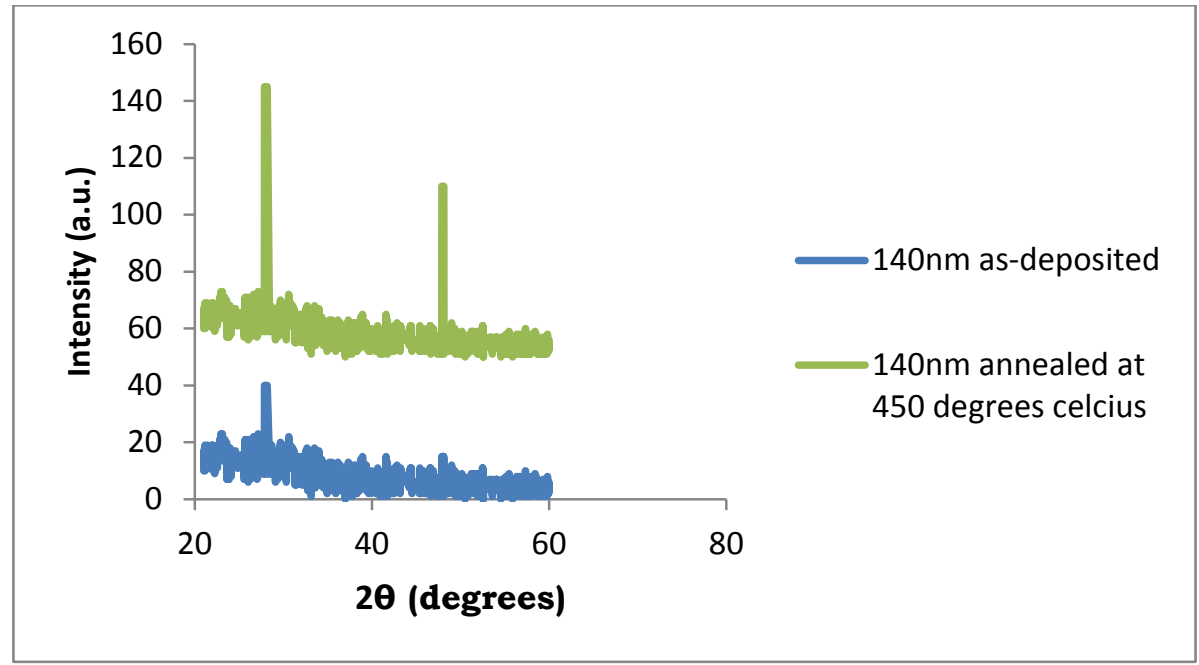

Figure 4.1(c): XRD pattern of CZTS thin film deposited at 140nm

Figure 4.1(d) shows the XRD pattern of CZTS thin film deposited at film thickness of 180nm. In the as-deposited sample, the broad hump ranging from $20^{\circ}$ and $28^{\circ}$ corresponds to the mixed amorphous and crystalline nature having short range periodicity of mixed ternary and quaternary phases of CuZnS or CZTS materials. Upon heat treatment (annealing at $450^{\circ} \mathrm{C}$ ), the broad hump disappeared and the peaks exhibited at about $28^{\circ}$ and $48^{\circ}$ respectively corresponds to (112) and (220) planes of tetragonal kesterite structure (JCPDS Card no. 26-0575). The transfer of one phase to another phase depends on the formation enthalpy of the particular phase [14].

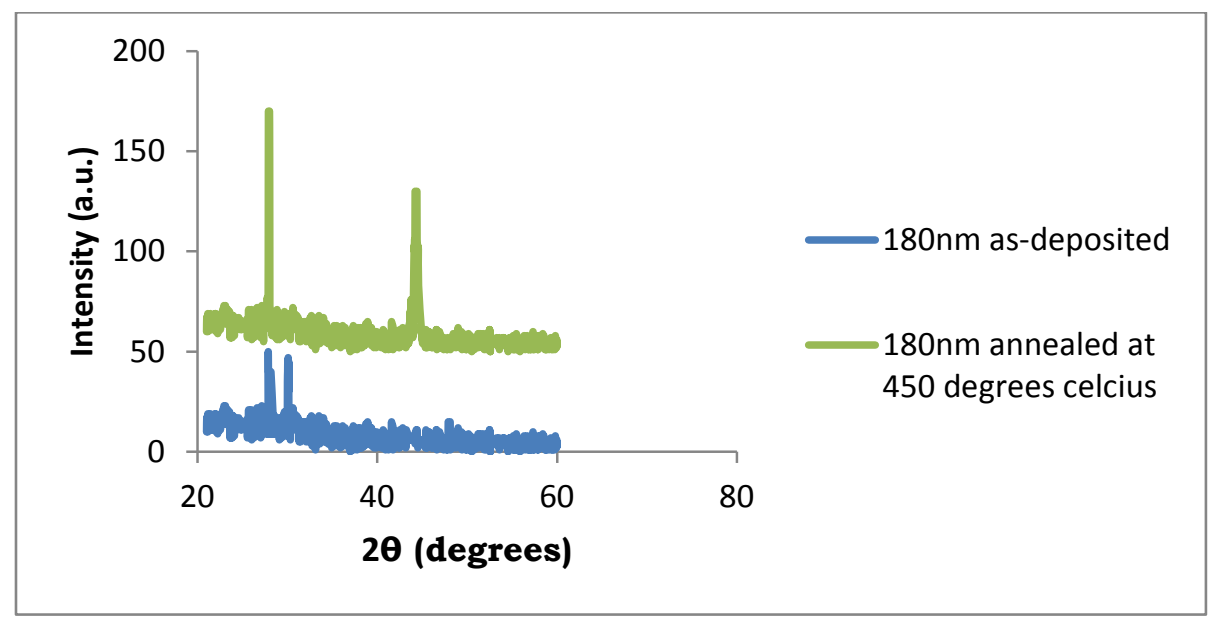

Figure 4.1(d): XRD pattern of CZTS thin film deposited at $180 \mathrm{~nm}$

The XRD patterns in all the annealed samples do not contain peaks corresponding to any secondary phases or constituent elements, which confirmed that the prepared film is homogeneous and single phase. The dominant peak observed at $2 \theta \approx 28^{0}$ is due to the reflection from a plane with preferred orientation (112). However, the diffraction peaks becomes stronger with increase in film thickness. The lattice parameters were calculated to be $a=5.4290 \mathrm{~A}$ and $\mathrm{c}=10.849 \mathrm{~A}$ using plane spacing equation and are in good agreement with the reported lattice parameters $\mathrm{a}=5.427 \mathrm{~A}$ and c=10.848A (JCPDS, card no. 20-0575). The crystallite size of the film was calculated using the Debye Sherer's equation. The average crystallite size of $\mathrm{Cu}_{2} \mathrm{ZnSnS}_{4}$ thin film was estimated to be $100 \mathrm{~nm}$. These observations suggest that the grown CZTS film has elemental concentration close to stoichiometric ratio. CZTS being a quaternary compound, it is difficult to control the compositional ratio close to stoichiometry. Thus, it requires additional control over synthesis parameters to obtain the desired phase of the material. However, the film with elemental composition close to expected stoichiometric ratio was achieved with RF -Magnetron sputtering in this work. Thus, a detailed analysis of the XRD data showed that the grown CZTS thin film has crystallized in kesterite structure with preferred orientation along the (112) plane.

Table 4.1 display the lattice parameter for the eight samples all grown at a substrate temperature of $100^{\circ} \mathrm{C}$. It was observed from the table that, for all the films, the grain size is lower in the annealed samples and thus, exhibit the most crystallinity. 
This is due to the small grains coalesced together during annealing to produce larger grains. Also, from the table, it can be seen that the dislocation density and the micro strain values are not affected by change in film thickness. The calculated values of dislocation density and micro strain are close to those reported by Shorr et al., 2011. It can also be observed that the FWHM is narrow in as-deposited samples than the annealed samples implying that thin films become more crystalline when annealed.

\subsection{CONCLUSIONS}

CZTS thin films have been successfully grown on corning glass substrate by RF-magnetron sputtering. The X-ray diffraction patterns of the CZTS $\left(\mathrm{Cu}_{2} \mathrm{ZnSnS}_{4}\right)$ thin films reveal that the film has crystallized in tetragonal type kesterite structure with space group. The peaks present in the XRD pattern indicate that the film has good crystalline nature and has strong preferred orientation along the (112) plane. All the peaks observed in the XRD pattern have been accounted for kesterite structure, which shows the absence of additional phases such as elemental, binary or ternary systems in the grown film. Therefore, the structural, properties of the grown thin films have showed that CZTS thin film is suitable for solar cell applications.

Table 4.1: Calculated structural parameters of CZTS thin films deposited at substrate temp. of $100^{\circ} \mathrm{C}$

\begin{tabular}{|c|c|c|c|c|c|c|}
\hline S/No & $\begin{array}{c}\text { Sample } \\
\text { Thickness } \\
\text { (nm) }\end{array}$ & $\begin{array}{l}\text { Annealing } \\
\text { Temp. } \\
\left({ }^{\circ} \mathrm{C}\right)\end{array}$ & $\begin{array}{c}\text { Grain } \\
\text { Size } \\
\text { D (nm) }\end{array}$ & $\begin{array}{l}\text { Micro } \\
\text { Strain } \\
\square \times 10^{-3}\end{array}$ & $\begin{array}{c}\text { Dislocation } \\
\text { Density } \\
\delta \times 10^{-14}\end{array}$ & $\begin{array}{c}\text { FWHM } \\
\text { B(degrees) }\end{array}$ \\
\hline 1. & 45 & As-dep & 0.373 & 0.0905 & 7.19 & 0.410 \\
\hline 2. & 45 & 450 & 0.307 & 0.1103 & 10.64 & 0.500 \\
\hline 3. & 90 & As-dep & 0.373 & 0.0905 & 7.19 & 0.410 \\
\hline 4. & 90 & 450 & 0.307 & 0.1103 & 10.64 & 0.500 \\
\hline 5. & 140 & As-dep & 0.373 & 0.0905 & 7.19 & 0.410 \\
\hline 6. & 140 & 450 & 0.307 & 0.1103 & 10.64 & 0.500 \\
\hline 7. & 180 & As-dep & 0.373 & 0.0905 & 7.19 & 0.410 \\
\hline 8. & 180 & 450 & 0.307 & 0.1103 & 10.64 & 0.500 \\
\hline
\end{tabular}

\section{REERENCES}

1. International Energy Agency (2014). Summit -New Trends in Solar Cell Applications- Amsterdam:10

2. U.S. Energy Information Administration(2013), International Energy Outlook -U.S. Department of Energy, Washington, DC.

3. U.S. Energy Information Administration(2011), International Energy Outlook -U.S. Department of Energy, Washington, DC.

4. Li JB, Chawla V and Clemens BM (2012) Investigating the Role of Grain Boundaries in CZTS and CZTSSe Thin Film Solar Cells with Scanning Probe Microscopy. Advanced Materials, 24: 6, 720-723

5. Acquire media (2011) Handbook of Photovoltaic Science and Engineering. Wiley, 2nd Edtion-11.

6. Business week (2011) Intergovernmental Panel on Climate Change:The IPCC Special Report on Renewable Energy Sources and Climate Change Mitigation (AUGUST), Geneva. 20-21

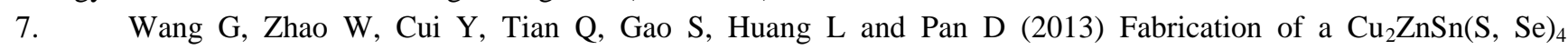
Photovoltaic Device by a Low Toxicity Solution Process. ACS Applied Mater Interfaces, 5, 10042-10047

8. Jiang $\mathrm{M}$ and $\mathrm{Yan} \mathrm{X},(2013) \mathrm{Cu}_{2} \mathrm{Zn} \mathrm{SnS}_{4}$ thin film solar cells present status and future prospects, INTECH (opensourcelopenminds), 5

9. Illican, I.,Caglar, Y. and Caglar M. (2008). Structural, Morphological and Optical Properties of CuAlS 2 thin films deposited by Spray Pyrolisis. Journal of Optoelectronics and Advanced Material 10: 10. 
10. Sarma K., Sarma, R. and H.L. Das (2008). Structural Characterization of Thermally Evaporated CdSe thin Films. Chalcogenide Letters. 5: 153-163

11. Tanaka, T, Yoshida A, Saiki D, Saito K, Guo, Q, Nisho M, and Yamaguchi T (2010). Thin solid films 518, 529533

12. Lakhe MG, Bhand GR, Londhe PU, Rohom AB and Chaure NB (2016) Electrochemical Synthesis and Characterization of $\mathrm{Cu}_{2} \mathrm{ZnSnS}_{4}$ Thin Films. Journal of Material Science and Engineering 5:2 pp 261

13. Arun Khalkar, Kwang-Soo Lim, Seong-Man Yu, Shashikant P. and Ji-Beom Yoo (2013) Effect of Growth Parameters and Annealing Atmosphere on the properties of $\mathrm{Cu}_{2} \mathrm{ZnSnS}_{4}$ Thin films Deposited by Co-sputtering. International journal of Photoenergy $\mathrm{pp} 7$

14. Shurr T, Tajima S and Ito T (2009) Enhancement of Conversion Efficiency of $\mathrm{Cu}_{2} \mathrm{ZnSnS}_{4}$ Thin Film Solar Cells by Improvement of Sulfurization Conditions. Applied Physics Express 6, 6:062301 